\title{
Experimental Observation on the Pure Torsional Ratchetting of Polycarbonate Polymer at Room Temperature
}

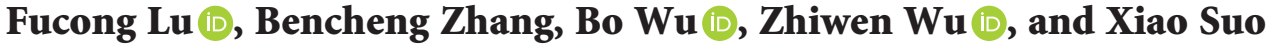 \\ College of Civil Engineering and Architecture, Key Laboratory of Disaster Prevention and \\ Structural Safety of Ministry of Education, Guangxi Key Laboratory of Disaster Prevention and Engineering Safety, \\ Guangxi University, Nanning 530004, China
}

Correspondence should be addressed to Bo Wu; wubo@gxu.edu.cn

Received 12 November 2019; Accepted 26 February 2020; Published 26 March 2020

Academic Editor: Aniello Riccio

Copyright (c) 2020 Fucong Lu et al. This is an open access article distributed under the Creative Commons Attribution License, which permits unrestricted use, distribution, and reproduction in any medium, provided the original work is properly cited.

\begin{abstract}
The stress-controlled pure torsional cyclic tests are carried out to investigate the torsional ratchetting of polycarbonate (PC) polymer at room temperature. The effects of applied shear mean stress, stress amplitude, stress rate, peak stress hold, and stress history on the torsional ratchetting are discussed. The shear strain of tubular specimen is measured by a noncontact digital image correlation (DIC) apparatus. The results show that the torsional ratchetting of the polymer obviously depends on the applied shear stress level, stress rate, and peak stress hold; the shear ratchetting strain and its rate increase with the increasing mean stress, stress amplitude, and peak stress hold time and with the decreasing stress rate. Moreover, the torsional ratchetting depends on the stress history. A higher stress level cyclic loading history restrains the evolution of torsional ratchetting in the subsequent lower stress level cyclic loading, while the lower stress level cyclic loading history promotes the torsional ratchetting of the subsequent higher level cyclic loading.
\end{abstract}

\section{Introduction}

Polycarbonate (PC) polymer is widely used in various engineering structures, such as pressure vessels, automotive components, aerospace structures, military equipment, and civil engineering due to its excellent mechanical properties [1-5]. These engineering structures are usually subjected to a cyclic loading, with ratchetting (produced in the case of asymmetric stress-controlled cyclic deformation) and fatigue failure as the main failure modes. Therefore, it is very important for the design and safety evaluation of engineering structures to systematically evaluate the cyclic deformation behavior of such materials.

Traditionally, most engineering structures are made of metallic materials, and the researches on cyclic deformation behavior of materials mainly focus on metallic materials. Therefore, there have been many experimental and theoretical researches on cyclic deformation behavior of metallic materials, which can be referred to the literature review [6]. However, experimental and theoretical studies on cyclic deformation behavior of polymeric materials are still lacking. In recent years, due to the more and more extensive applications of polymeric materials in engineering, many researchers began to pay attention to the study of cyclic deformation behavior of these materials; for example, Chen et al. [7] investigated the influence of humidity on cyclic deformation behavior and cyclic heat generation of polyamide- 6 polymer. The results show that the change of humidity will not change the inherent cyclic softening/ hardening features of the material but obviously affects the degree of cyclic softening/hardening of the material; Liu et al. [8] discussed the effects of stress cycling on the physical aging of polycarbonate polymer; Holopainen et al. [9] proposed a model to simulate the ratchetting and fatigue interaction behavior of polycarbonate polymer in the framework of continuum mechanics; Hughes et al. [10] experimentally studied the fatigue behavior of polycarbonate polymers and proposed a multistage fatigue model to evaluate the crack evolution; Zhang et al. [11] proposed a method to accelerate ratchetting testing based on the time- 
temperature equivalence principle and verified the effectiveness of the proposed method with the uniaxial ratchetting test of polycarbonate polymer; Li et al. [12] studied the effect of cyclic deformation on the mechanical properties of polycarbonate polymer. The results reveal that, during the initiation of fatigue damage, the fracture toughness of the material decreases with the increasing number of cyclic loadings significantly, while the yield strength is almost unaffected; Fang et al. [13] conducted cyclic deformation experiments on polycarbonate (PC) and polycarbonate/acrylonitrile-butadiene-styrene $(\mathrm{PC} / \mathrm{ABS})$ and discussed the effects of cyclic loading on the uniaxial tensile properties of the two kinds of materials. The results demonstrate that the yield and fracture stress of PC/ABS are lower than those of $\mathrm{PC}$, while the reduction of fracture stress and strain caused by cyclic loading of PC is greater than that of PC/ABS; Chen et al. [14-16] systematically studied the deformation behavior of ultrahigh molecular weight polyethylene under stress-controlled cyclic loading and discussed the ratchetting behavior under uniaxial and nonproportional multiaxial loading, respectively. Based on the experimental results, a cyclic constitutive model considering the nonproportional multiaxis effect was proposed; Yang et al. $[17,18]$ conducted a series of stress-controlled cyclic loading experiments on polyamide- 6 material, discussed the ratchetting-fatigue interaction of the material, and found that ratchetting strain has a harmful effect on the fatigue life of the material; Tong et al. [19] conducted uniaxial stress and strain-controlled cyclic loading tests on solid propellant polymer composites and discussed the influence of thermal generation on the material properties; our previous papers $[4,5]$ studied the uniaxial and multiaxial ratchetting of polycarbonate polymer and discussed the influence of temperature on the uniaxial ratchetting and the effect of loading path on the multiaxial ratchetting of the material. From the existing literatures, it can be concluded that the ratchetting of polymers is greatly affected by temperature, humidity, loading rate, loading path, average stress, stress amplitude, and other factors due to the significant viscoelasticity of the materials. At the same time, the ratchetting has some harmful effects on the material's fatigue life. However, the existing literatures mainly focus on the uniaxial ratchetting of polymers and seldom pay attention to multiaxis ones. At present, there is no special paper to systematically study the pure torsional ratchetting of polymeric materials.

Therefore, in this paper, a systematic experimental study on the pure torsional cyclic deformation of polycarbonate polymer is carried out, and the torsional ratchetting and cyclic softening/hardening of the material under pure torsional cyclic loading were discussed. These experimental results, as a supplement to the results of our previous papers $[4,5]$, will provide a basis for the establishment of the constitutive model of the material and facilitate the application of the material in engineering.

\section{Experimental Procedures}

The material used in this experimental study is consistent with that of our previous article [4]. The raw material is an extruded PC polymer bar from BAYER Makrolon ${ }^{\circledR}$. The thin-walled hollow specimen is obtained from the original rod through mechanical processing, and its dimensions are shown in Figure 1. Such a specimen design with very short gauge length $(5 \mathrm{~mm})$ can effectively prevent buckling instability during torsion. Using the material mechanics formula, the relationship between equivalent shear stress $\tau_{e}$ and the applied torque $T$ is given as follows:

$$
\tau_{e}=\sqrt{3} \tau=\sqrt{3} \frac{16 T}{\pi\left(D^{2}-d^{2}\right)(D+d)},
$$

where $D$ and $d$ are the outer and inner diameters of tubular specimens, respectively.

The tests were performed on an MTS electrohydraulic servo universal material testing system (MTS858-BIONIX). The strain measuring method by traditional extensometer will inevitably cause damage to the surface of specimen $[4,20]$ and cannot be used for specimen with a very short gauge length. Therefore, to solve the problem, based on the proposed scheme of our previous work [4], a noncontact 3D digital image correlation (DIC) system (ARAMIS 5M, GOM mhH Ltd., Germany) was used in this experimental study to capture the full-field strain evolution of the specimen's surface during testing. The experimental setup is shown in Figure 2. This paper mainly studies the pure torsional ratchetting of the $\mathrm{PC}$, which is the evolution of shear strain accumulation under asymmetric shear stress-controlled cyclic loading. However, prior to the ratchetting tests, some additional tests were carried out to investigate the basic mechanical properties and the cyclic softening/hardening features of the PC material. These additional tests include a series of monotonic torsion, torsional shear creep, and torsional angle-controlled cyclic testings. The results of these basic testings are very helpful to the selection of stress levels in subsequent ratchetting tests. The prescribed load cases of torsional angle-controlled cyclic testing are listed in Table 1 and those of the stress-controlled cyclic testing are listed in Table 2. It should be noted that, due to the independence of DIC and MTS testing systems, the shear strain measured by the DIC system cannot real-time feedback to the MTS control system, so only a torsional angle-controlled cyclic test, rather than real shear strain-controlled one, is carried out to study the cyclic softening/hardening properties of the $\mathrm{PC}$ in this work.

To illustrate the torsional ratchetting more clearly and compare it with the existing uniaxial one more directly, the equivalent torsional ratchetting strain $\gamma_{\mathrm{er}}$ defined as follows is used in the data analysis:

$$
\gamma_{\mathrm{er}}=\frac{\left(\gamma_{e \max }+\gamma_{\mathrm{emin}}\right)}{2},
$$

where $\gamma_{e \max }$ is the maximum equivalent shear strain (i.e., $\gamma_{e}=\gamma / \sqrt{3}$, and $\gamma$ is shear strain) in each cycle; $\gamma_{e m i n}$ is the minimum one. Torsional ratchetting strain rate is defined as the increment of equivalent torsional ratchetting strain $\gamma_{\mathrm{er}}$ per cycle. To conveniently analyze the shear strain recovery after creep tests, percentage of residual strain recovery (PRSR) defined as follows is used in this work: 


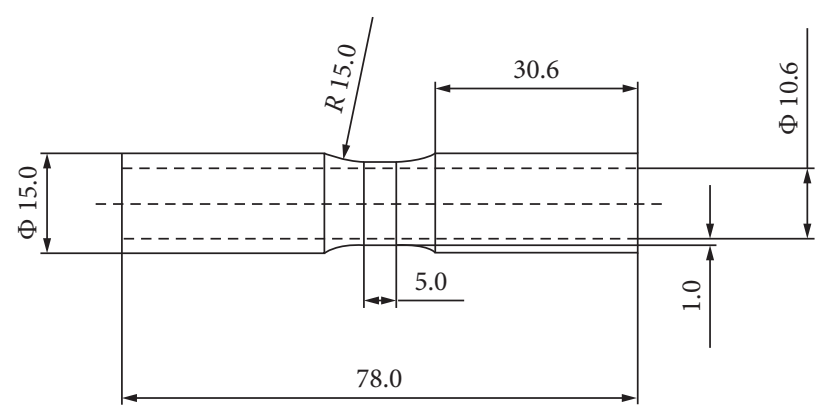

FIGURE 1: Shape and sizes of thin-walled hollow specimen (unit: $\mathrm{mm}$ ).

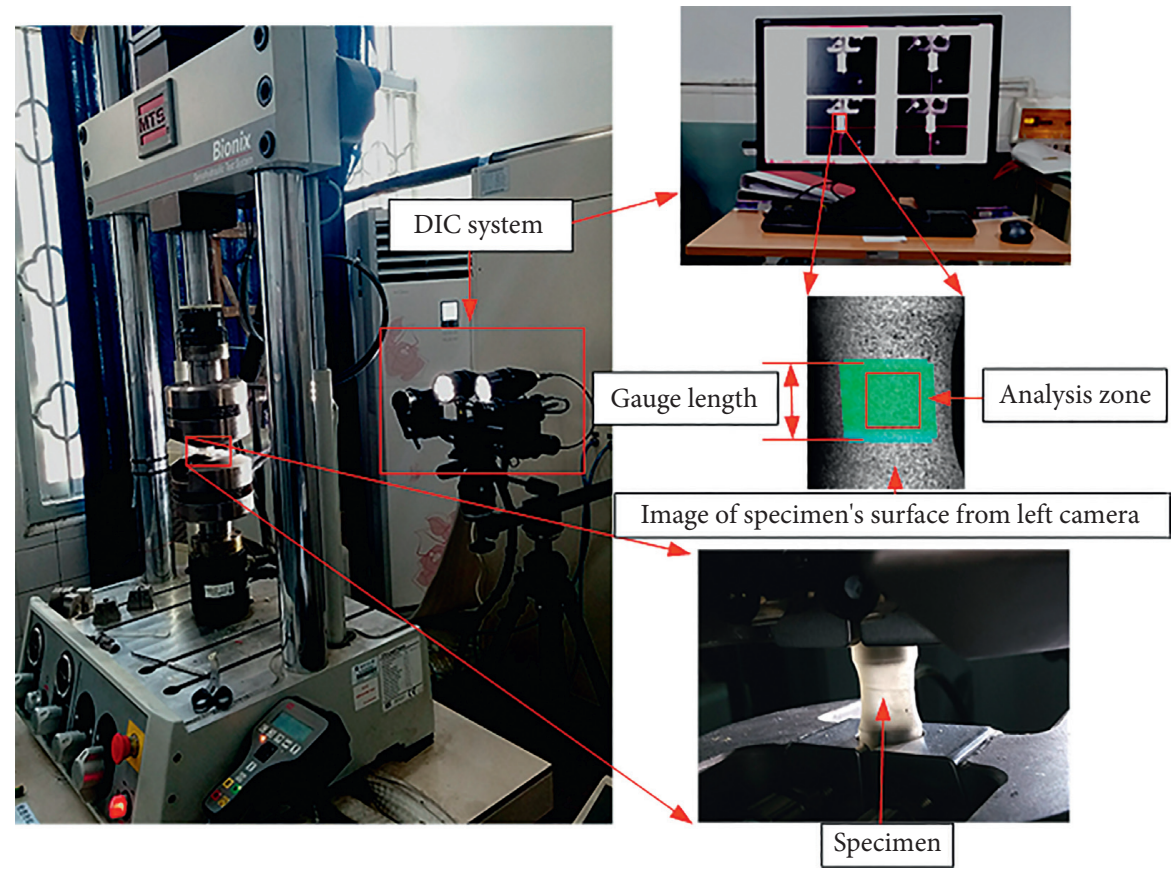

Figure 2: Experimental setup: test machine and DIC system.

TABLE 1: Torsional angle-controlled cyclic loading cases.

\begin{tabular}{lcccc}
\hline Loading cases & Mean torsional angle $\left({ }^{\circ}\right)$ & Torsional angle amplitude $\left(^{\circ}\right)$ & Torsional angle rate $\left({ }^{\circ} / \mathrm{s}\right)$ & Number of cycles $(N)$ \\
\hline Stra1 & 0 & 9 & 5 & 200 \\
Stra2 & 0 & 8 & 5 & 200 \\
Stra3 & 0 & 7 & 5 & 200 \\
Stra4 & 0 & 7 & 0.5 & 200 \\
\hline
\end{tabular}

$$
\operatorname{PRSR}=\left(\gamma_{\mathrm{er} 0}-\gamma_{\mathrm{erT}}\right) / \gamma_{\mathrm{er} 0} \times 100 \%,
$$

where $\gamma_{\mathrm{er} 0}$ is the original residual shear strain and $\gamma_{\mathrm{erT}}$ is the current residual shear strain after the recovering for $T$ seconds.

\section{Results and Discussion}

3.1. Monotonic Torsion. Figure 3(a) illustrates the equivalent shear stress-strain curves obtained in the monotonic torsional tests under the torsional angle-controlled condition and at load rates of $0.1 \%$ and $2 \%$, respectively; the evolution of equivalent shear strain field in the analysis zone of specimen is also provided in the figure for the load case at lower load rate (i.e., $0.1^{\circ} / \mathrm{s}$ ). It is seen in Figure $3(\mathrm{~b})$ that, similar to the monotonic tensile stress-strain curves discussed in $[21,22]$ for other PCs, apparent nonlinear stressstrain response occurs before the macroscopic yielding and the pure torsional deformation behavior of the $\mathrm{PC}$ also is rate-dependent; however, the stress-strain responses at yielding point and beyond the yielding (i.e., at the stage of softening) in monotonic torsional tests are different from 
TABLE 2: Stress-controlled cyclic loading cases ${ }^{1}$.

\begin{tabular}{lcccc}
\hline Loading cases & Mean stress $(\mathrm{MPa})$ & Stress amplitude $(\mathrm{MPa})$ & Peak hold time $(\mathrm{s})$ & Stress rate $(\mathrm{MPa} / \mathrm{s})$ \\
\hline Stre1 & 15 & 30 & 0 & 1 \\
Stre2 & 25 & 30 & 60 & 1 \\
Stre3 & 25 & 30 & 0 & 1 \\
Stre4 & 35 & 30 & 10 & 1 \\
Stre5 & 35 & 30 & 60 & 1 \\
Stre6 & 35 & 30 & 0 & 1 \\
Stre7 & 35 & 30 & 0 & 1 \\
Stre8 & 30 & 15 & 0 & 1 \\
Stre9 & 30 & 25 & 0 & 1 \\
Stre10 & 30 & 35 & 0 & 1 \\
Stre11 & $25 \longrightarrow 35$ & 30 & 0 & 1 \\
Stre12 & $35 \longrightarrow 25$ & 30 & 0 & 1 \\
Stre13 & 30 & $35 \longrightarrow 25 \longrightarrow 35$ & 0 & 1 \\
Stre14 & 30 & $25 \longrightarrow 35 \longrightarrow 25$ & $\longrightarrow$ & 1 \\
\hline
\end{tabular}

${ }^{1}$ Note: 50 cycles for every load case without stress history (i.e., Stre1-Stre10) and 90 cycles for that with stress history (i.e., Stre11-Stre14).

those in the tensile ones. In the monotonic torsional cases (Figure 3(a)), a yielding plateau is observed, and the strain softening after the yielding is not as remarkable as that in the monotonic tensile ones (Figure 3(b)). From the evolution of shear strain field shown in Figure 3(a), it is also seen that the equivalent shear strain increases homogeneously from 0 to $10 \%$ (i.e., from point $A$ to point $D$ ) and the average equivalent shear strain in the analysis zone here can represent precisely the shear strain of the specimen within the gauge length; however, when the equivalent shear strain increases further, for example, after point $E$, the equivalent shear strain field becomes heterogeneous as shown in Figure 3(a). The heterogeneous distribution of shear strain leads to a quick bulking of the specimen. Therefore, in the following ratchetting tests, the applied stress level is prescribed to be smaller than the yielding stress of the PC, so that the resultant equivalent torsional ratchetting strain is within the range of $10 \%$.

3.2. Creep Followed by Recovery. Creep tests followed by recovery ones (simplified as creep-recovery tests) are conducted to reveal the viscosity of the PC, and three specimens are, respectively, loaded to different equivalent shear stress levels (i.e., 35, 55, and $65 \mathrm{MPa}$ ) at an equivalent shear stress rate of $1 \mathrm{MPa} / \mathrm{s}$ and then held for 2 hours (i.e., creeping) and then finally unloaded to zero stress and held at zero stress for 1 hour (i.e., recovering). The obtained creep and recovery curves are shown in Figure 4. It can be seen that the equivalent shear strain increases quickly at the beginning of creep tests, but its increasing rate (i.e., creep rate) decreases with the creep time; after certain creep times, the creep rate becomes constant, and a stable creep occurs as shown in Figure 4(a). Generally, a higher shear stress results in a larger torsional creep strain, which is similar to the tensile creep of the PCs conducted by Xi et al. [21] and Jiang et al. [22]. Moreover, Figure 4(a) illustrates that the residual shear strain after the unloading from the creep tests will be partially recovered during the subsequent zero stress hold due to the viscoelasticity of the PC; however, the recovery rate at the first beginning of recovering is much higher than that at the end of recovering. From the evolution curves of the PRSR (i.e., percentage of residual strain recovery defined in equation (3)) shown in Figure 4(b), it is concluded that the PRSR obtained in the creep-recovery tests with higher creep shear stress is smaller than that with smaller creep shear stress, since more unrecoverable viscoplastic deformation is produced in the creep-recovery tests with higher creep shear stress. Due to the influence of environmental noise, the test results will inevitably fluctuate. The smaller the strain value is measured, the smaller the strain value is and the more fluctuation is.

3.3. Torsion Angle-Controlled Cyclic Test. Test results of the torsional angle-control cyclic loading cases are shown in Figure 5. The evolution of a typical stress-strain curve (Figure 5(a)) demonstrates that the stress-strain hysteresis loop is very narrow and hardly changes during the cyclic test. Figure 5(b) shows the evolution of the response stress amplitudes during cyclic tests with several different loading angle amplitudes and two loading rates. It is clearly seen that all the curves are almost horizontal, meaning that the response stress amplitudes do not change by the number of loading cycles. It is concluded that PC is a kind of cyclic stable material, which shows no softening/hardening features. At the same time, it can be clearly observed from Figure 5(b) that, under the same torsional angle amplitude $\left( \pm 7^{\circ}\right)$ but with different loading rate $\left(0.5^{\circ} / \mathrm{s}\right.$ and $\left.5^{\circ} / \mathrm{s}\right)$, the response shear stress amplitude with higher loading rate is much higher than that with the lower loading rate, indicating that the material shows an obvious rate-dependent mechanical behavior.

3.4. Torsional Ratchetting and Its Time-Dependence. The torsional ratchetting of the PC is investigated in this subsection by asymmetrical shear stress-controlled cyclic tests, and the dependence of ratchetting on the applied shear stress level, stress history, and stress rate and peak stress hold (i.e., time-dependent ratchetting) are discussed. 


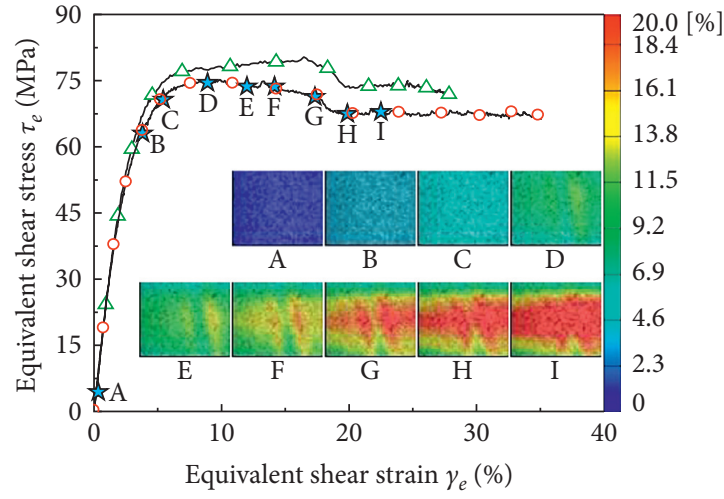

Load rate:

$\triangle \sim 2 \%$

$-0.1 \%$

(a)

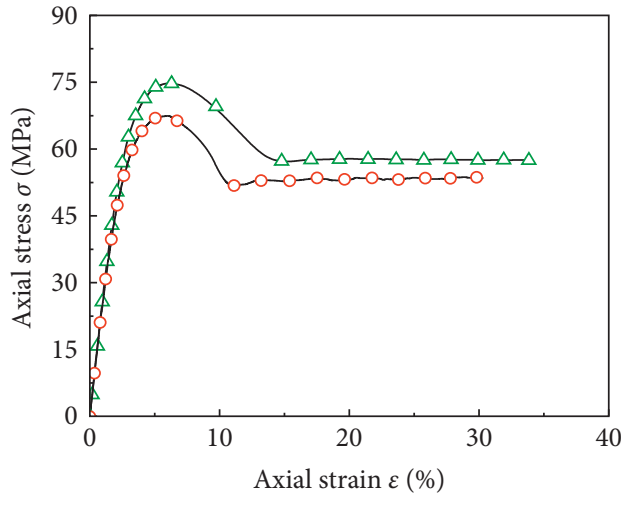

Load rate:

$\rightarrow-0.5 \mathrm{~mm} / \mathrm{s}$

$\multimap 0.01 \mathrm{~mm} / \mathrm{s}$

Figure 3: Stress-strain curves at different load rates and in (a) monotonic pure torsional test and (b) monotonic tensile test.

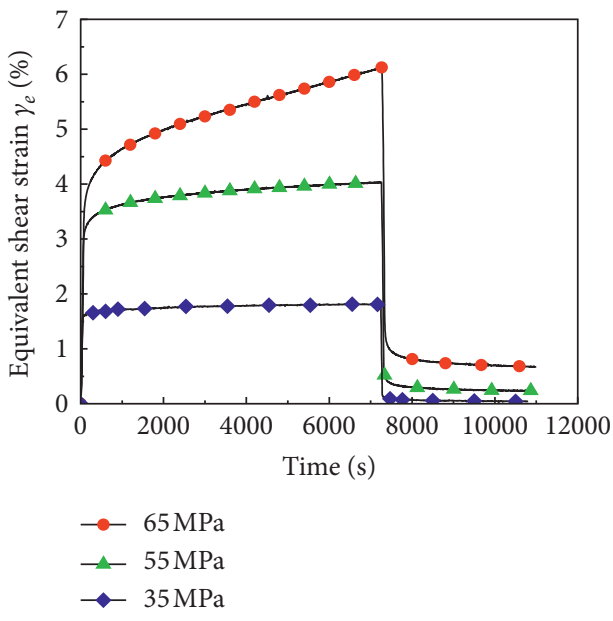

(a)

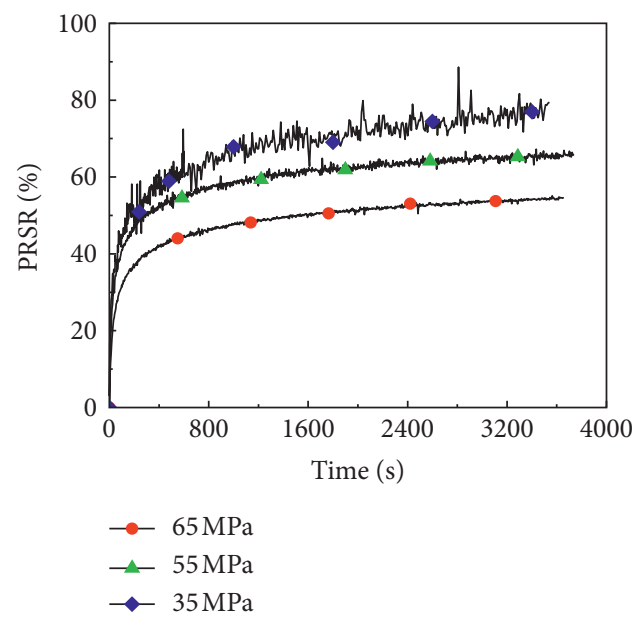

(b)

FIGURE 4: Results of creep-recovering tests: (a) curves of equivalent shear strain versus testing time; (b) curves of percentage of residual strain recovery (PRSR) versus recovering time.

3.4.1. Effect of Shear Stress Level on Pure Torsional Ratchetting. Figure 6 shows the torsional ratchetting and the evolution of ratchetting strain $\gamma_{\mathrm{er}}$ (defined by equation (2)) obtained in the shear stress-controlled cyclic tests with identical equivalent shear stress amplitude of $\pm 30 \mathrm{MPa}$ and different mean shear stresses (i.e., 15, 25, and $35 \mathrm{MPa}$ ) and at stress rate of $1 \mathrm{MPa} / \mathrm{s}$. It is seen from Figure 6 that apparent ratchetting, that is, cyclically accumulated inelastic deformation, occurs in the pure torsional stress-controlled cyclic tests of the PC too; and the ratchetting strain $\gamma_{\mathrm{er}}$ increases with the number of cycles but the ratchetting strain rate (i.e., the increment of $\gamma_{\text {er }}$ per cycle) decreases gradually during the cyclic loading. After certain cycles, a stable ratchetting at a constant rate is reached, as shown in Figure 6(b). Moreover, it is concluded that the torsional ratchetting of the PC depends greatly on the applied mean shear stress, and the ratchetting strain increases with the increasing mean shear stress as shown in Figure 6(b). From the results obtained in the cyclic tests with identical mean shear stress of $30 \mathrm{MPa}$ and different shear stress amplitudes (i.e., 15, 25, and $35 \mathrm{MPa}$ ), as shown in Figure 7 , it is concluded that the torsional ratchetting depends greatly on the applied shear stress amplitude too, and the ratchetting strain $\gamma_{\mathrm{er}}$ increases with the increasing stress amplitude. Furthermore, comparison of the results shown in Figures 6(b) and 7 demonstrates that the effect of mean shear stress on the torsional ratchetting is more significant than that of shear stress amplitude. It should be noted that since the applied stress level (the maximum peak equivalent shear stress is $65 \mathrm{MPa}$ ) in the prescribed ratchetting tests is lower than the 


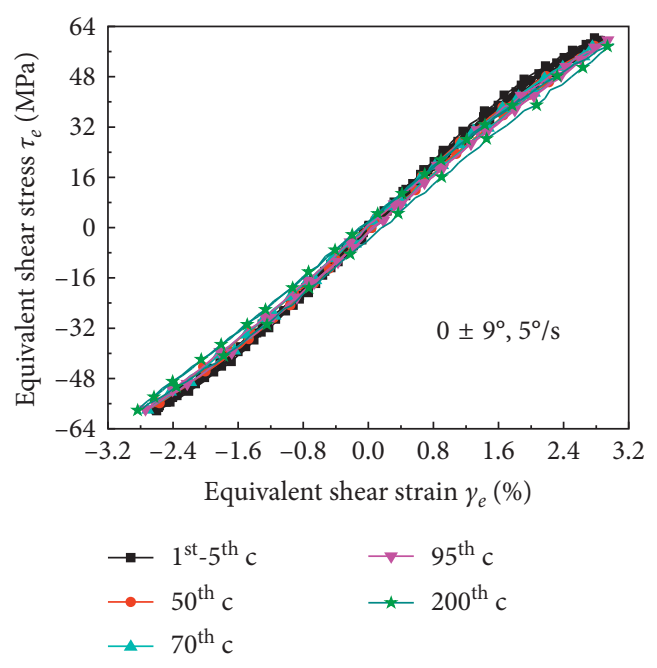

(a)

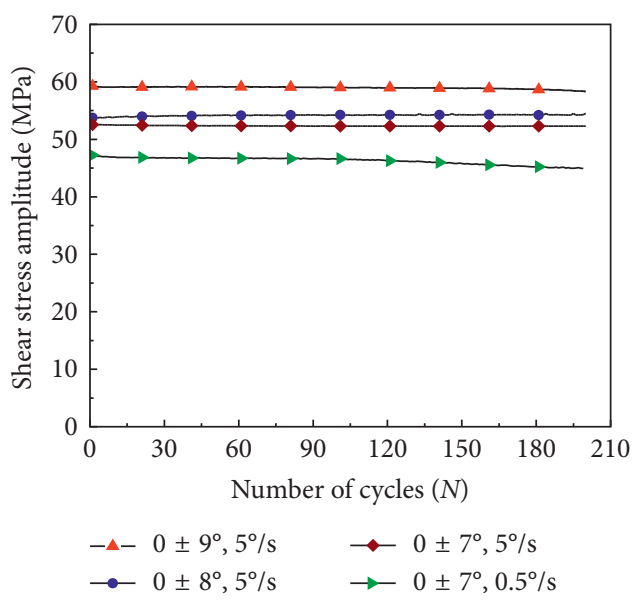

(b)

Figure 5: Angle-controlled cyclic testing results: (a) stress-strain hysteresis curves with load case of angle amplitude of $\pm 9^{\circ}$ and rate of $5^{\circ} /$; (b) respondent shear stress amplitude versus the number of loading cycles under prescribed load cases.

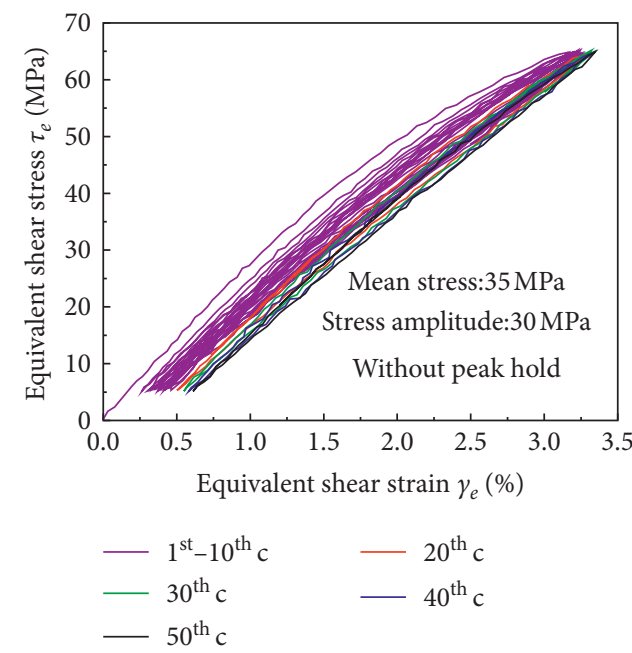

(a)

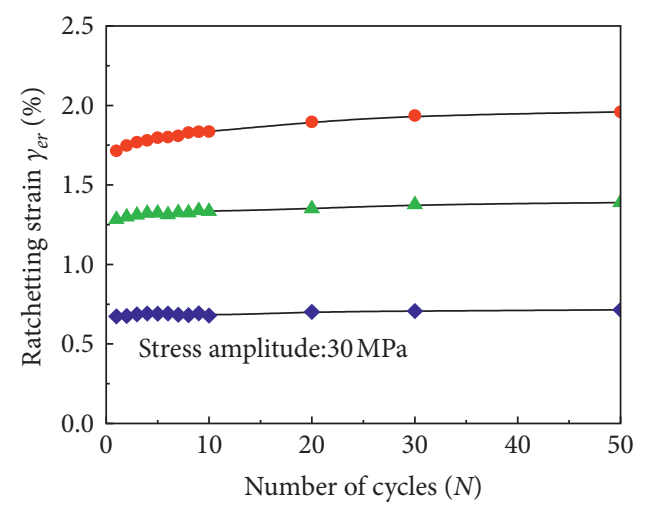

Mean stress:
$--35 \mathrm{MPa}$
$-\backsim 25 \mathrm{MPa}$
$-\bullet 15 \mathrm{MPa}$

(b)

Figure 6: Results of stress-controlled cyclic tests: (a) stress-strain hysteresis of stress level $35 \pm 30 \mathrm{MPa}$ and stress rate at $1 \mathrm{MPa} / \mathrm{s}$; (b) evolution ratchetting strain versus number of cycles with different mean stresses and the same shear stress amplitude.

corresponding yielding stress (i.e., about $75 \mathrm{MPa}$ ) and the pure torsional stress-strain responses of the PC at this stage of stress level are similar to those of monotonic tensile ones, in the sense of evolution rule, the torsional ratchetting of the PC discussed here is similar to the uniaxial ratchetting.

3.4.2. Effect of Stress History on Torsional Ratchetting. To study the effect of stress history on the torsional ratchetting of the PC, some tests are conducted with different loading histories. For the load cases with mean stress history, two kinds of mean stress histories with the sequences of high $\longrightarrow$ low $\longrightarrow$ high (i.e., $35 \longrightarrow 25 \longrightarrow 35 \mathrm{MPa)}$ and low $\longrightarrow$ high $\longrightarrow$ low (i.e., $25 \longrightarrow 35 \longrightarrow 25 \mathrm{MPa}$ ) are used.
The stress rate is $1 \mathrm{MPa} / \mathrm{s}$. The results are shown in Figures 8 and 9. It is observed that, after experiencing certain cycles with a higher mean shear stress, the torsional ratchetting in the subsequent cyclic loading with a lower mean shear stress hardly occurs, and the ratchetting rate is almost zero as shown in Figure 9(a); however, if the applied mean shear stress is reraised to a higher one again after certain cycles, new torsional ratchetting occurs. It implies that the previous loading history with higher mean shear stress will significantly restrain the torsional ratchetting in the subsequent cyclic loading with lower mean shear stress, which is similar to that of metal material observed by Kang et al. [23]. For the load case with low $\longrightarrow$ high $\longrightarrow$ low mean shear stress sequence, it is seen that the previous cyclic loading with lower 


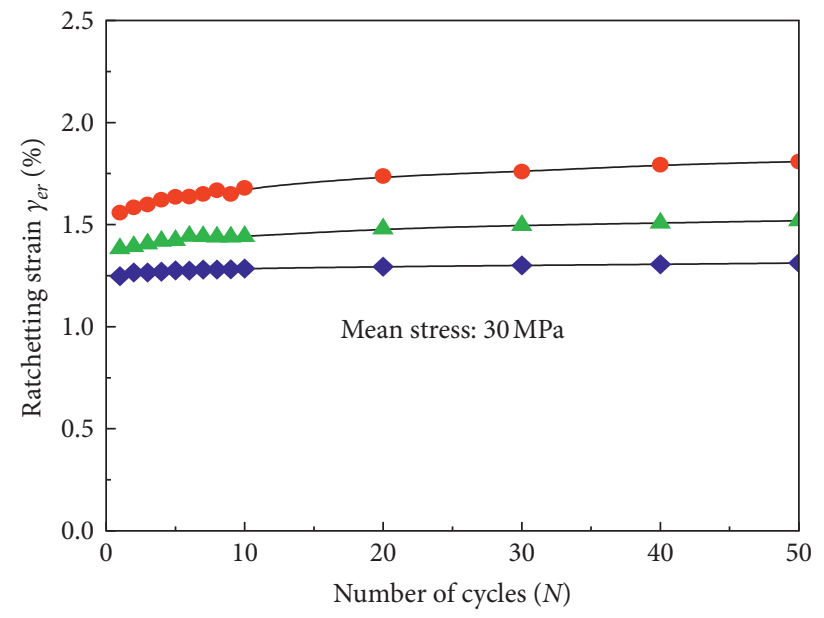

Stress amplitude:

- $-35 \mathrm{MPa}$

$-25 \mathrm{MPa}$

$-15 \mathrm{MPa}$

Figure 7: Evolution of ratchetting strain versus number of cycles obtained in the cyclic tests with different stress amplitudes and an identical mean shear stress.

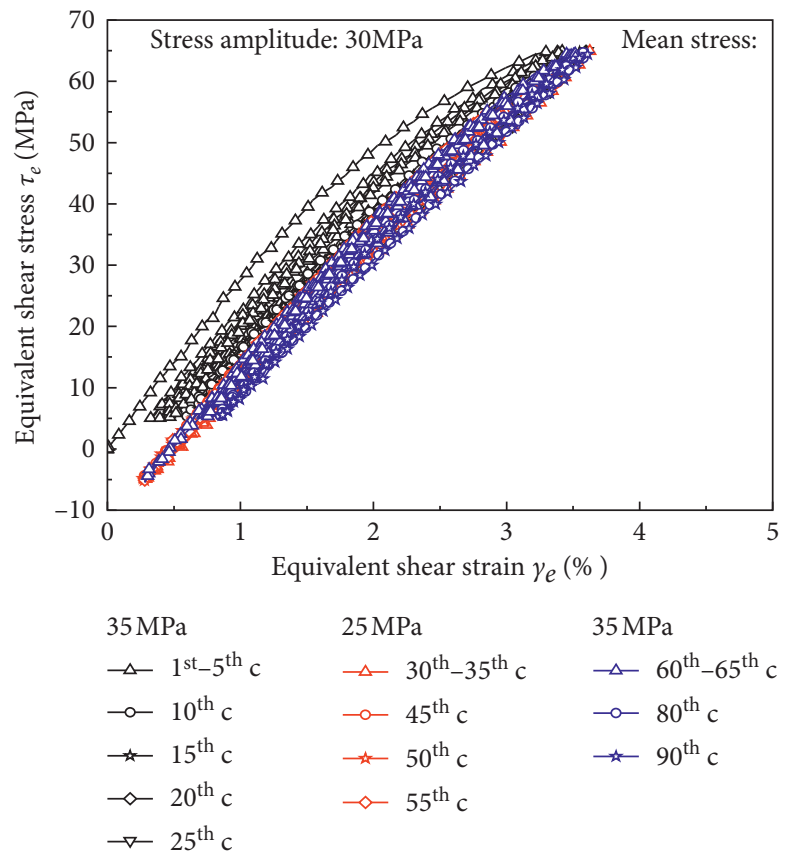

FiguRE 8: Stress-strain curve obtained in the stress-controlled cyclic test with a mean stress history (i.e., $35 \longrightarrow 25 \longrightarrow 35 \mathrm{MPa}$ ) and at stress rate of $1 \mathrm{MPa} / \mathrm{s}$.

mean shear stress does not restrain the torsional ratchetting of the PC in the subsequent cyclic loading with higher mean shear stress, as shown in Figure 9(b). Comparing the results for the load parts of $35 \pm 30 \mathrm{MPa}$ in the prescribed two load cases, as shown in Figures 9(a) and 9(b), it is seen that the torsional ratchetting in the load part experiencing a previous cyclic loading with lower mean shear stress is more remarkable than that without previous loading history. It means that, for the PC, the previous cyclic loading with lower mean shear stress will promote the development of torsional ratchetting in the subsequent cyclic loading with higher mean shear stress to a certain extent.

From the results obtained from the tests with shear stress amplitude history and shown in Figures 10(a) and 10(b), it is seen that the stress amplitude history also has significant influence on the torsional ratchetting of the $\mathrm{PC}$, which is similar to that of mean shear stress history shown in Figures 9(a) and 9(b). 


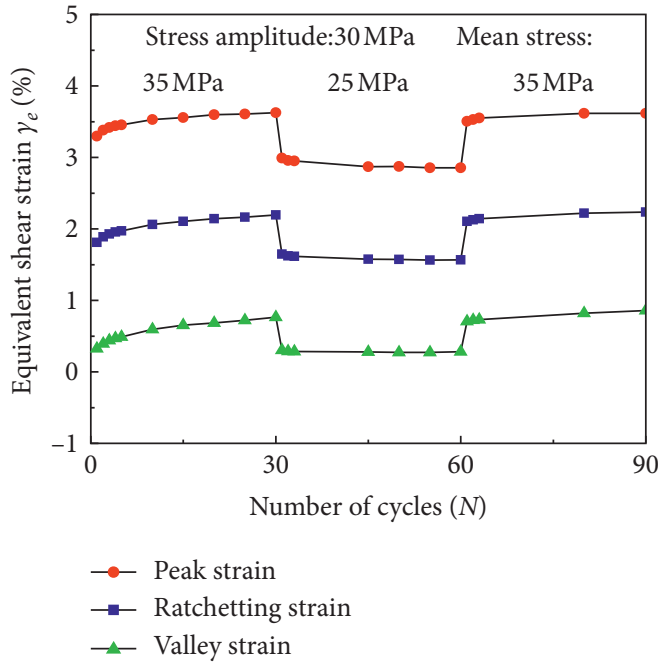

(a)

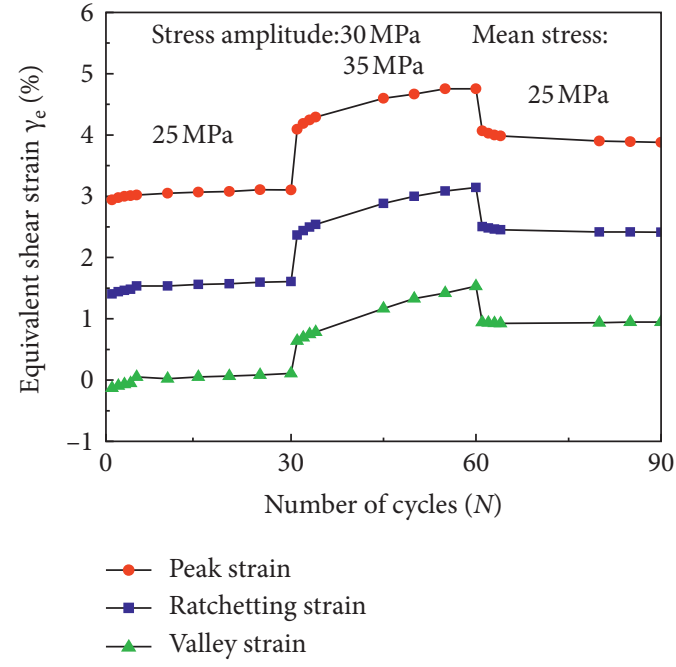

(b)

FIGURE 9: Curves of peak, valley, and ratchetting strains versus number of cycles obtained in stress-controlled cyclic tests at stress rate of $1 \mathrm{MPa} / \mathrm{s}$ and with various mean stress histories: (a) $35 \longrightarrow 25 \longrightarrow 35 \mathrm{MPa}$; (b) $25 \longrightarrow 35 \longrightarrow 25 \mathrm{MPa}$.

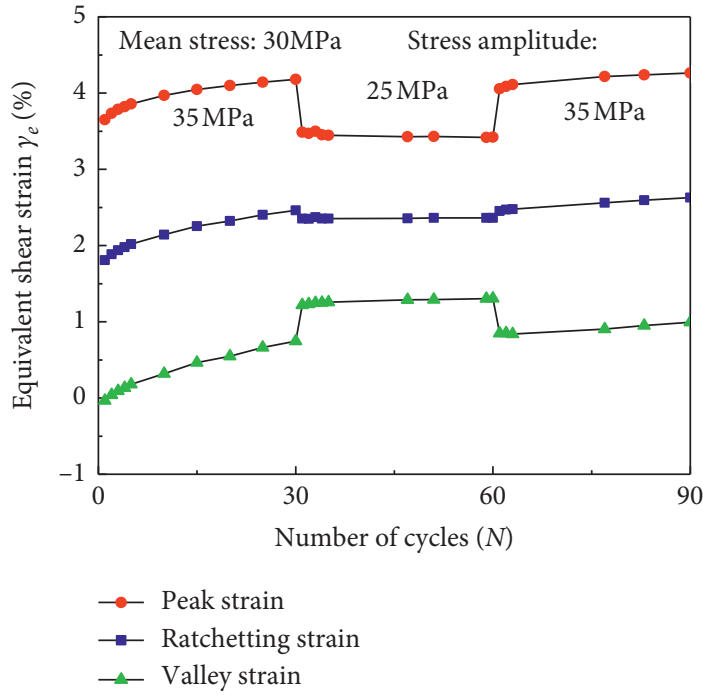

(a)

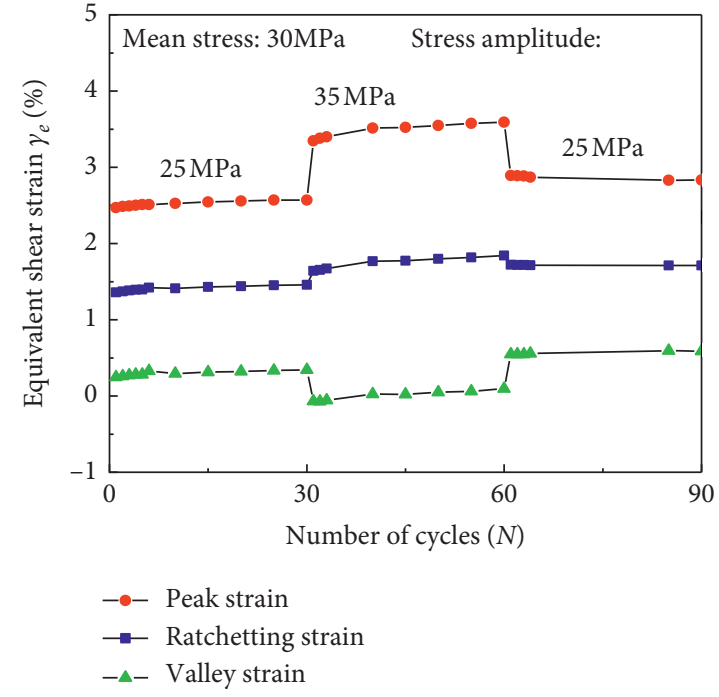

(b)

Figure 10: Curves of peak, valley, and ratchetting strains versus number of cycles obtained in stress-controlled cyclic tests at stress rete of $1 \mathrm{MPa}$ /s and with various stress amplitude histories: (a) $35 \longrightarrow 25 \longrightarrow 35 \mathrm{MPa}$; (b) $25 \longrightarrow 35 \longrightarrow 25 \mathrm{MPa}$.

To sum up, the shear stress history has remarkable influence on the torsional ratchetting of the PC: a higher stress level cyclic loading history will significantly restrain the evolution of torsional ratchetting in the subsequent lower stress level cyclic loading, while the lower stress level cyclic loading history can promote the torsional ratchetting of the subsequent higher level cyclic loading. The results hint that constructing a reasonable cyclic constitutive model of the PC should consider the effects of loading history.

3.4.3. Time-Dependent Torsional Ratchetting. Due to the obvious viscosity of the $\mathrm{PC}$, as revealed by monotonic pure torsion, torsional creep, and torsional angle-controlled cyclic tests (Figures 3-5), the time-dependence of mechanical properties of the material is significant. Investigating the time-dependence of torsional ratchetting of the PC is needed. The results shown in Figures 11-13 are obtained from the torsional ratchetting tests with or without peak stress hold and at different shear stress rates, respectively. The details of prescribed loading conditions can be referred to Table 2 .

It is concluded from Figures 11 and 12 that the torsional ratchetting is more remarkable in the load case with a certain peak shear stress hold than that without any peak stress hold, and the ratchetting strain $\gamma_{\mathrm{er}}$ increases with the increases of 


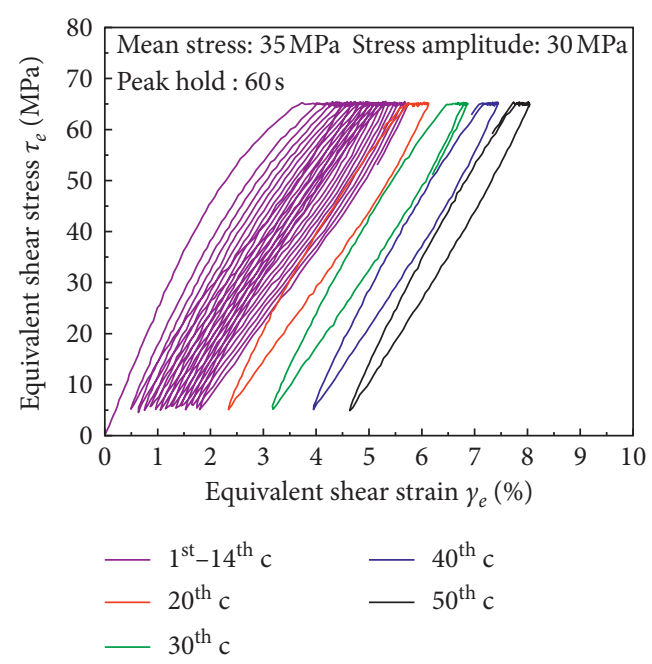

(a)

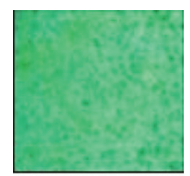

$1^{\text {st }} \mathrm{c}$

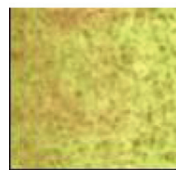

$20^{\text {th }} \mathrm{c}$

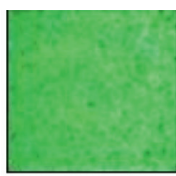

$5^{\text {th }} \mathrm{c}$

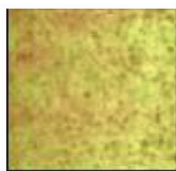

$30^{\text {th }} \mathrm{c}$

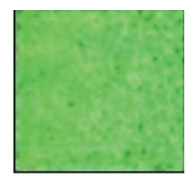

$10^{\text {th }} \mathrm{c}$

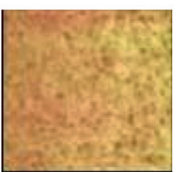

$40^{\text {th }} \mathrm{c}$

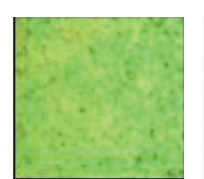

$15^{\text {th }} \mathrm{c}$

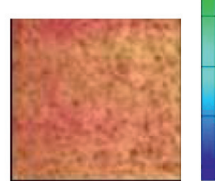

$50^{\text {th }} \mathrm{c}$

Figure 11: Results obtained in the stress-controlled cyclic test with $35 \pm 30 \mathrm{MPa}$ and peak hold for $60 \mathrm{~s}$, at $1 \mathrm{MPa} / \mathrm{s}$ : (a) equivalent shear stress-strain curves; (b) distribution of equivalent shear strain immediately at the end of peak stress hold and its evolution during the cyclic loading.

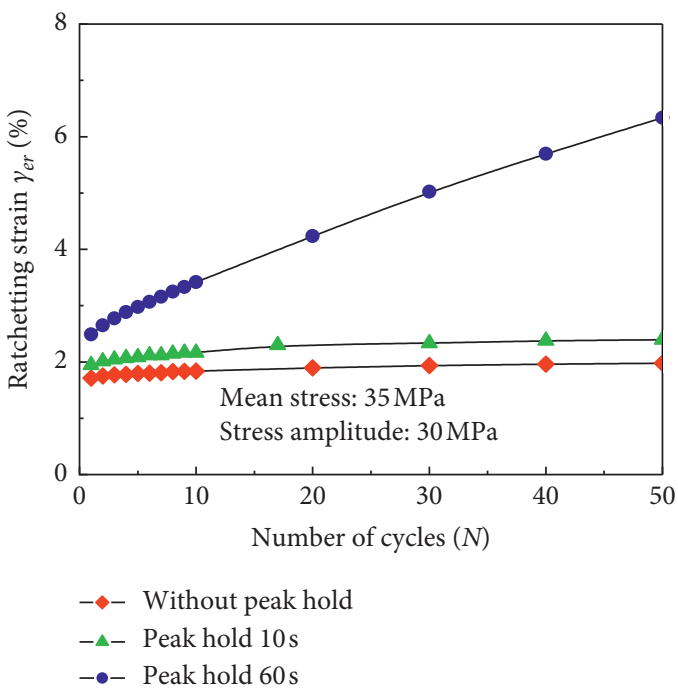

(a)

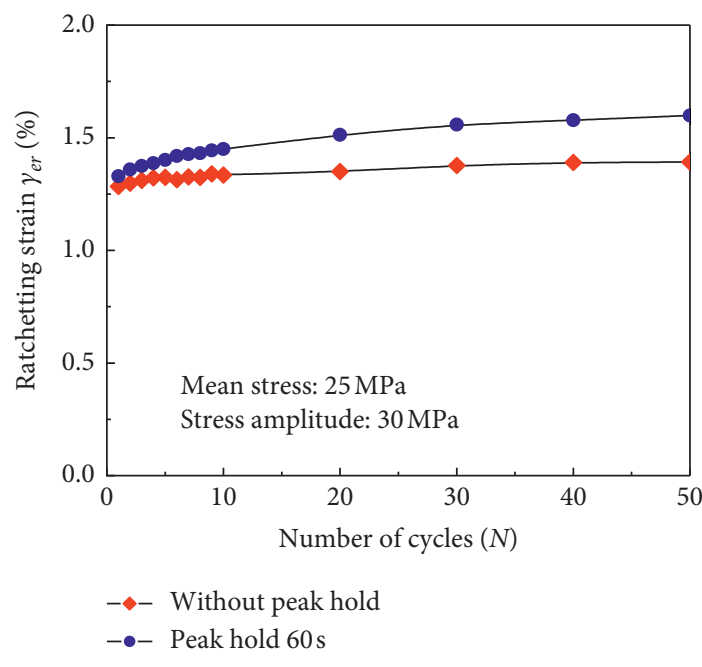

(b)

FIGURE 12: Curves of ratchetting strain versus number of cycles obtained in cyclic tests with various peak hold times and different stress levels, and at $1 \mathrm{MPa} / \mathrm{s}$ : (a) $35 \pm 30 \mathrm{MPa}$; (b) $25 \pm 30 \mathrm{MPa}$.

hold times at peak stress point and values of applied peak shear stresses. The increase of ratchetting strain caused by the peak stress hold can be attributed to the creep strain produced in the period of stress hold due to the obvious viscosity of the PC. The strain field in the analysis zone of the specimen is still homogeneous for the test with highest peak shear stress and longest hold time as shown in Figure 11(b), and no buckling of tubular specimen occurs. From Figure 13, it is seen that the torsional ratchetting depends greatly on the applied stress rate, and the ratchetting strain $\gamma_{\mathrm{er}}$ produced in the cyclic test at higher stress rate is smaller than that at lower one.

To sum up, the torsional ratchetting of the PC is greatly time-dependent at room temperature, similar to that observed for the uniaxial ratchetting of another PC [21].

It should be noted that the torsional ratchetting of the PC presented in the cyclic tests with stress levels lower than the PC's shear yielding stress is similar to the uniaxial one in the sense of evolution rule. However, the investigation of 


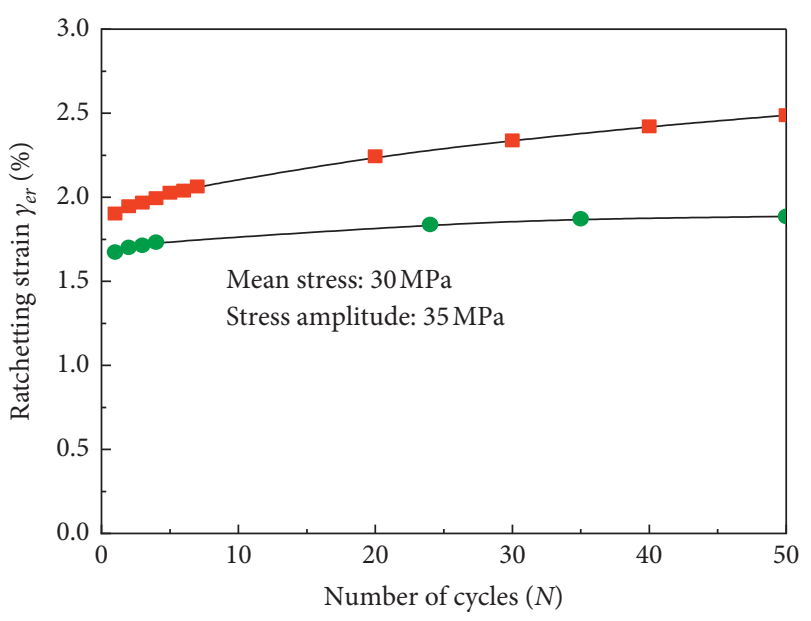

Stress rate:

$-\square-1 \mathrm{MPa} / \mathrm{s}$

$-10 \mathrm{MPa} / \mathrm{s}$

Figure 13: Curves of ratchetting strain versus number of cycles obtained in cyclic tests with $30 \pm 35 \mathrm{MPa}$ and at two stress rates.

torsional ratchetting is still very helpful to realize the combined tension-torsion multiaxial ratchetting of the $\mathrm{PC}$, and then the obtained data in this work are very useful. Moreover, different from the previous researches $[4,5]$, the effect of loading history on the torsional ratchetting of the $\mathrm{PC}$ is investigated in this work.

\section{Conclusions}

From the observations to the torsional ratchetting of the PC, the following conclusions are obtained:

(1) The PC exhibits a significant rate-dependent torsional deformation, and the shear yield stress of the material increases significantly with the increase of loading rate. Different from the monotonic tensile test, no significant strain softening is observed beyond the yielding of the PC in the monotonic torsional one.

(2) The PC presents a significant torsional creep deformation due to its viscosity. More percentage of residual strain is recoverable after the creeping with lower peak stress than that with higher one.

(3) No cyclic hardening/softening feature is observed in the torsional angle-controlled cyclic test, and the PC can be assumed to be a cyclic stable material.

(4) Apparent torsional ratchetting occurs in the shear stress-controlled cyclic tests of the PC. The torsional ratchetting depends greatly on the shear stress level, stress history, stress rate, and peak stress hold. The larger ratchetting strain is produced in the cyclic test with higher stress level, lower stress rate, and longer peak stress hold. The prior loading history with higher stress level remarkably restrains the occurrence of torsional ratchetting in the subsequent cyclic loading with lower one, while the prior loading history with lower stress level will promote the development of torsional ratchetting in the subsequent cyclic loading with a higher one.

\section{Data Availability}

The datasets used and analyzed during the current study are available from the corresponding author upon reasonable request.

\section{Conflicts of Interest}

The authors declare that there are no conflicts of interest regarding the publication of this paper.

\section{Acknowledgments}

The authors gratefully acknowledge the financial support by the projects of National Natural Science Foundation of China (51678164, 51478118, and 51808136) and Guangxi Natural Science Foundation Program (2018GXNSFDA138009 and 2018GXNSFBA281199).

\section{References}

[1] C. Koomson, S. E. Zeltmann, and N. Gupta, "Strain rate sensitivity of polycarbonate and vinyl ester from dynamic mechanical analysis experiments," Advanced Composites and Hybrid Materials, vol. 1, no. 2, pp. 341-346, 2018.

[2] Y. Xu, T. Gao, J. Wang, and W. Zhang, "Experimentation and modeling of the tension behavior of polycarbonate at high strain rates," Polymers, vol. 8, no. 3, p. 63, 2016.

[3] G. Kang and Q. Kan, Cyclic Plasticity of Engineering Materials: Experiments and Models, John Wiley \& Sons, Hoboken, NJ, USA, 2017.

[4] F. Lu, G. Kang, Y. Zhu, C. Xi, and H. Jiang, "Experimental observation on multiaxial ratchetting of polycarbonate polymer at room temperature," Polymer Testing, vol. 50, pp. 135-144, 2016.

[5] F. Lu, G. Kang, H. Jiang, J. Zhang, and Y. Liu, "Experimental studies on the uniaxial ratchetting of polycarbonate polymer at different temperatures," Polymer Testing, vol. 39, pp. 92100, 2014.

[6] G. Kang, "Ratchetting: recent progresses in phenomenon observation, constitutive modeling and application," International Journal of Fatigue, vol. 30, no. 8, pp. 1448-1472, 2008.

[7] K. Chen, G. Kang, F. Lu, J. Chen, and H. Jiang, "Effect of relative humidity on uniaxial cyclic softening/hardening and intrinsic heat generation of polyamide- 6 polymer," Polymer Testing, vol. 56, pp. 19-28, 2016.

[8] L. B. Liu, A. F. Yee, and D. W. Gidley, "Effect of cyclic stress on enthalpy relaxation in polycarbonate," Journal of Polymer Science Part B: Polymer Physics, vol. 30, no. 3, pp. 221-230, 1992.

[9] S. Holopainen, T. Barriere, G. Cheng, and R. Kouhia, "Continuum approach for modeling fatigue in amorphous glassy polymers. Applications to the investigation of damageratcheting interaction in polycarbonate," International Journal of Plasticity, vol. 91, pp. 109-133, 2017.

[10] J. M. Hughes, M. Lugo, J. L. Bouvard, T. McIntyre, and M. F. Horstemeyer, "Cyclic behavior and modeling of small 
fatigue cracks of a polycarbonate polymer," International Journal of Fatigue, vol. 99, pp. 78-86, 2017.

[11] J. Zhang, H. Jiang, C. Jiang, G. Kang, and F. Lu, "Accelerated ratcheting testing of polycarbonate using the time-temperature-stress equivalence method," Polymer Testing, vol. 44, pp. 8-14, 2015.

[12] X. Li, H. A. Hristov, A. F. Yee, and D. W. Gidley, "Influence of cyclic fatigue on the mechanical properties of amorphous polycarbonate," Polymer, vol. 36, no. 4, pp. 759-765, 1995.

[13] Q.-Z. Fang, T. J. Wang, H. G. Beom, and H. M. Li, "Effect of cyclic loading on tensile properties of PC and PC/ABS," Polymer Degradation and Stability, vol. 93, no. 8, pp. 14221432, 2008.

[14] K. Chen, G. Kang, F. Lu, and H. Jiang, "Uniaxial cyclic deformation and internal heat production of ultra-high molecular weight polyethylene," Journal of Polymer Research, vol. 22, p. 217, 2015.

[15] K. Chen, G. Kang, F. Lu, J. Xu, and H. Jiang, "Temperaturedependent uniaxial ratchetting of ultra-high molecular weight polyethylene," Fatigue \& Fracture of Engineering Materials \& Structures, vol. 39, no. 7, pp. 839-849, 2016.

[16] K. Chen, G. Kang, C. Yu, H. Jiang, and H. J. Qi, "Nonproportional multiaxial ratchetting of ultrahigh molecular weight polyethylene polymer: experiments and constitutive model," Mechanics of Materials, vol. 112, pp. 76-87, 2017.

[17] J. Yang, G. Kang, K. Chen, and Q. Kan, "Experimental study on uniaxial ratchetting-fatigue interaction of polyamide-6," Polymer Testing, vol. 69, pp. 545-555, 2018.

[18] J. Yang, G. Kang, K. Chen, Q. Kan, and Y. Liu, "Experimental study on rate-dependent uniaxial whole-life ratchetting and fatigue behavior of polyamide 6," International Journal of Fatigue, vol. 132, Article ID 105402, 2020.

[19] X. Tong, X. Chen, J.-S. Xu, Y. Zheng, and S.-J. Zhi, "The heat build-up of a polymer matrix composite under cyclic loading: experimental assessment and numerical simulation," International Journal of Fatigue, vol. 116, pp. 323-333, 2018.

[20] G. Tao and Z. Xia, "A non-contact real-time strain measurement and control system for multiaxial cyclic/fatigue tests of polymer materials by digital image correlation method," Polymer Testing, vol. 24, no. 7, pp. 844-855, 2005.

[21] C. Xi, G. Kang, F. Lu, J. Zhang, and H. Jiang, "An experimental study on uniaxial ratcheting of polycarbonate polymers with different molecular weights," Materials \& Design, vol. 67, pp. 644-648, 2015.

[22] H. Jiang, J. Zhang, G. Kang, C. Xi, C. Jiang, and Y. Liu, “A test procedure for separating viscous recovery and accumulated unrecoverable deformation of polymer under cyclic loading," Polymer Testing, vol. 32, no. 8, pp. 1445-1451, 2013.

[23] G. Kang, Q. Gao, and X. Yang, "Uniaxial cyclic ratcheting and plastic flow properties of SS304 stainless steel at room and elevated temperatures," Mechanics of Materials, vol. 34, no. 3, pp. 145-159, 2002. 